Review

\title{
Suspended-core Fibers for Sensing Applications
}

\author{
Orlando FRAZÃO ${ }^{1}$, Ricardo M. SILVA ${ }^{1,2}$, Marta S. FERREIRA ${ }^{1,2}$, \\ José L. SANTOS ${ }^{1,2}$, and António B. LOBO RIBEIRO ${ }^{3}$ \\ ${ }^{1}$ INESC Porto, Rua do Campo Alegre 687, 4169-007 Porto, Portugal \\ ${ }^{2}$ Faculty of Sciences of University of Porto, Rua do Campo Alegre, 687, 4169-007 Porto, Portugal \\ ${ }^{3}$ Faculty of Health Sciences, University Fernando Pessoa, Rua Carlos da Maia, 296, 4200-150 Porto, Portugal \\ *Corresponding author: Orlando FRAZÃOＥ-mail: ofrazao@inescporto.pt
}

\begin{abstract}
A brief review on suspended-core fibers for sensing applications is presented. A historical overview over the previous ten years about this special designed microstructure optical fiber is described. This fiber presents attractive optical properties for chemical/biological or gas measurement, but it can be further explored for alternative sensing solutions, namely, in-fiber interferometers based on the suspended-core or suspended-multi-core fiber, for physical parameter monitoring.
\end{abstract}

Keywords: Optical fiber sensor, suspended core fiber, interferometry

\section{Introduction}

In 2001, Monro et al. [1] proposed a new design for a microstructured optical fiber, called "suspended-core fiber" (SCF). It was a single-mode fiber with a very small core dimension and presented $17 \%$ of the mode energy located in the air for a wavelength operation at $1550 \mathrm{~nm}$. In 2007, Webb et al. [2] demonstrated a simple fabrication method of the SCF. The technique consisted in defining the holes through mechanical drilling of the preform, which was a significantly quicker and more straightforward approach to the customary stacking method. During the draw, the shape of the holes was manipulated so that the final fiber design approximated that of an air-suspended rod with three fine struts supporting the core. This technique allowed the production of new geometries for the SCF to be applied to several solutions, notably in gas sensing and also for Raman spectroscopy and fluorescence applications. Nevertheless, in 2005, a different physical geometry with the enhanced hydrostatic pressure sensitivity was proposed [3]. An SCF was fabricated with the triangular cladding shape. Nowadays, these types of microstructured fibers are used for optical sensing [4] and also for nonlinear optical effects, namely for supercontinuum sources [5, 6] or fiber lasers [7]. Chemical or biological sensing and gas sensing are areas where the use of this type of fibers can be of the great asset, due to the high overlap factor of the evanescence field [4]. The SCF fibers present some disadvantages when being spliced to a single mode fiber (SMF). For instance, when subjected to the gas, its diffusion time is slow. Some authors solved this issue by exposing the silica fiber core with a femtosecond laser [8] or by using polymeric fibers [9]. Moreover, the small cross area section (nanoscale cores) allows this design to present high sensitivity to chemical [10] and physical parameters [11]. Lately, in 2008, the plasmonics was studied in SCFs and was used as a refractometer for aqueous

Received: 15 January 2012 / Revised version: 1 March 2012

(C) The Author(s) 2012. This article is published with open access at Springerlink.com 
analytes. Typically, these geometries are fabricated in the pure silica and present low sensitivity to the temperature [11]. Over the previous years, different materials have been used to enhance other properties, in particular, nonlinear optical effects [12]. The interferometry is also another type of a possible application. The intermodal (high core dimension), birefringence (asymmetric core), double core and multicore are intrinsic characteristics of the in-fiber interferometry that can be created by properly designing the core [13, 14]. Recently, a suspended-core polymer fiber for terahertz guiding was reported for imaging and sensing applications [15].

This review intends to describe the recent advances using SCF in optical sensing over the areas where it has been exploited. Special focus will be given to in-fiber interferometers for physical parameters sensing.

\section{Brief review}

Suspended-core fibers have been used in several applications. In this review, all research works analyzed were divided in four main groups, according to the applications: physical parameters, gas and liquid sensing, fluorescence and Raman scattering, and finally biological parameters.

\subsection{Physical parameters}

In 2005, MacPherson et al. [3] reported the first sensor using a microstructure optical fiber (MOF) presenting the enhanced sensitivity to the pressure. The geometry of the microstructure fiber provided an intrinsic hydrostatic pressure sensitivity of the optical path length. The sensitivities achieved for pressure and temperature were $7.86 \mathrm{rad} / \mathrm{bar} / \mathrm{m}$ and $77 \mathrm{rad} /{ }^{\circ} \mathrm{C} / \mathrm{m}$, respectively.

In 2008, Huy et al. [16] developed a fiber Bragg grating(FBG)-based refractometer using a germanium doped core SCF. The geometric properties of the SCF have been optimized in order to improve the evanescent field overlapping with any medium inserted in the holes. An improvement in the sensitivity by two orders of magnitude compared to a six-hole photonic crystal fiber (PCF) was also demonstrated. The resolution of $3 \times 10^{-5}$ and $6 \times 10^{-6}$ was achieved for a mean refractive index value of 1.33 and 1.44, respectively. Later on, Hautakorpi et al. [17] presented a novel surface-plasmon-resonance sensor by coating the holes of an SCF with a low-index dielectric layer on the top of which a gold layer was deposited. With this type of sensor a resolution of $1 \times 10^{-4}$ was achieved for aqueous solutions. A Sagnac interferometer using a section of the SCF as the sensor for temperature-independent strain measurement was proposed by Frazão et al. [11]. The sensor was analyzed in two situations, with and without the coating. The strain sensitivity of the sensor was about $1.94 \mathrm{pm} / \mu \varepsilon$ for the two situations. Comparing epoxy/acrylate and pure silica, its Young modulus is around five times lower. The pure silica presents higher stiffness, therefore all deformation occurs in this region. The technology and some characteristics of the SCF manufactured with the core undoped and doped with germanium dioxide were investigated by Wojcik et al. [18]. In some fibers a silver layer was deposited, which enabled investigation of surface-plasmon-resonance in optical fiber sensing applications.

In 2009, the fundamental mode cutoff properties of germanium doped SCFs was investigated by Torres-Pieró et al. [19]. Filling the air-holes of the SCF with the liquid allowed the implementation of the wavelength- and amplitude-encoded temperature sensors. The cutoff wavelength changed with temperature and the thermo-optic coefficient of the liquid determined the sensitivity of the sensor. A sensitivity of $25 \mathrm{~nm} /{ }^{\circ} \mathrm{C}$ was obtained for temperature, and a simple amplitude interrogation for the sensing head was also implemented. Gauvreau et al. [20] presented a novel high numerical aperture multimode polymer fiber. The transmission performances were analyzed, offering a low propagation loss in the visible and enabling microfluidic applications. Frazão et al. [21] 
proposed a new geometry for a Fabry-Pérot (FP) structure using two types of SCF as the sensing element. The different sensing heads were characterized in respect to temperature and strain. In the same year, a refractometer using a hybrid structure combined the SCF in series with a section of the SMF was also proposed [22].

In 2010, a Sagnac fiber loop mirror (FLM) sensor based on an SCF was investigated by Frazão et al. [13]. Due to the mechanical stress in the geometry of the two cores, the refractive index difference between them was about $10^{-3}$. The difference was generated during the process of fabrication. The sensing element was characterized in torsion, temperature and strain. Applying the fast Fourier transform (FFT), a temperature- and strain-independent torsion sensor was attained. The sensitivity obtained was $1.2 \times 10^{-2} \mathrm{~dB} /$ degree. The same group proposed a new sensing head based on a Mach-Zehnder interferometer (MZI) using an SCF. Since the two cores have high birefringence, two discrete interferometers could be obtained [23]. Different sensitivities were achieved: $-2.56 \mathrm{~nm} \cdot \mathrm{m}$ and $-11.4 \mathrm{pm} /{ }^{\circ} \mathrm{C}$ for curvature and temperature, respectively. The matrix computing method was used in order to discriminate these two measurands. Later on, Pinto et al. [7] reported an interrogation system of an FP cavity through a dual wavelength Raman fiber laser. The cavity was formed by splicing an SCF to an SMF. The sensing system was able to generate two quadrature phase-shifted signals that allowed recovering the temperature change sensed by the FP cavity. The interrogation configuration permitted a passive and accurate temperature measurement.

In 2011, an all-fiber Mach-Zehnder interferometer configuration based on an SCF was described by Silva et al. [24]. The sensor presented different sensitivities to strain, curvature and temperature. Simultaneous measurement was demonstrated through the application of the matrix computing method to discriminate the three parameters. Jewart et al. [25] presented the simulation and experimental results of using an FBG inscribed in an SCF. Due to the fiber structure, the FGBs were more sensitive to the applied transverse load than the standard FBGs written in the SMF. With this characteristic it was possible to create an embedded fiber strain gauge that could simultaneously measure both directions of axial and transverse strains. A multicore SCF for simultaneous measurement of curvature and strain was proposed by Silva et al. [14]. Due to seven cores of the sensing head, the spectral response showed evidences of several interferences. The sensor presented different sensitivities to curvature and strain, allowing the discrimination of measurements using the matrix method.

In 2012, Bravo et al. [26] investigated a high sensitivity micro-displacement fiber sensor based on a Sagnac interferometer. An SCF section was used to create an FLM. High precision was obtained for a displacement of about $0.45 \mathrm{~m}$. Moreover, a high stability and insensitivity to temperature was shown. Aref et al. [27] reported two all-fiber interferometric configurations using an SCF. In the first configuration, an FP cavity was made with a length of SCF spliced between segments of the SMF and hollow-core fiber. An alternative sensing configuration was the insertion of the SCF in the output ports of a $3-\mathrm{dB}$ coupler forming an FLM. Pressure sensitivity and temperature response were measured to assess the cross sensitivity of these two measurands in both configurations.

\subsection{Gas and liquid sensing}

The first SCF based gas sensor was presented by Webb et al. [2] in 2007. A modal overlap higher than $29 \%$ at $1550 \mathrm{~nm}$ was achieved for a core diameter of $0.8 \mathrm{~m}$. Figure 1 presents the setup used to monitor the gas sensing. In the same year, Cordeiro et al. [28] presented alternative solutions to improve fluid sensing with an SCF. By inserting the sample through a lateral access to the fiber longitudinal holes, the fiber tips were free for optical handling and accessing. Euser et al. [29] demonstrated a 
quantitative broadband fiber chemical sensor in 2008. They exploited not only the fabrication processes but also the structural and optical characterization of a range of different fibers. Gas and liquid fiber sensors were characterized.

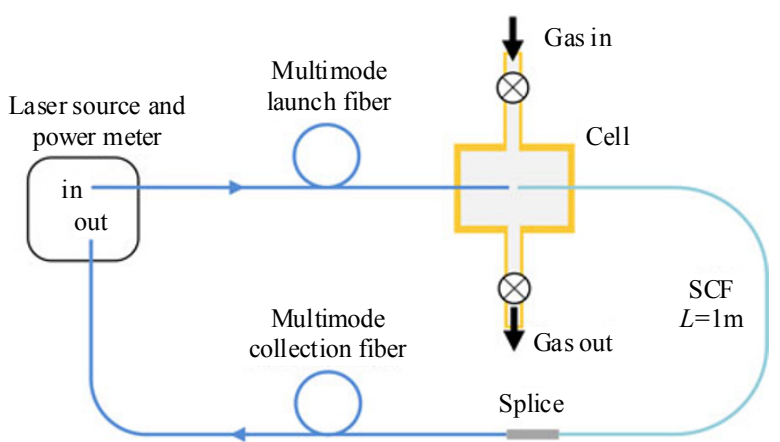

Fig. 1 Gas sensing schematic configuration proposed by Webb et al. [2].

In 2009, Lehmann et al. [30] compared the responses of the SCF and hollow core band-gap fibers for gas sensing in the near infrared. Various geometries of the SCF and hollow core band-gap fibers with different properties were tested, regarding their relative sensitivity and usable spectral bandwidth. The SCF proved to be better suited for the high gas concentration and/or for rather large sensor lengths. It was also stated that it could be a solution for condensed liquids with rather low refractive indices and particles, due to the robustness to the interferences of the light guiding in the SFC. In the same year, Selleri et al. [31] studied theoretically the electromagnetic field behavior of the guided modes of several SCFs and the behavior of the fundamental mode of a number of liquid-filled SCFs.

\subsection{Fluorescence and Raman scattering}

A generic model of excitation and fluorescence recapturing within the filed SCF with an arbitrary structure was presented by Afshar et al. [32] in 2007. They argued that, if the fabrication process was careful, fiber losses lower than $0.5 \mathrm{~dB} / \mathrm{m}$ could be achieved, thus highly-sensitivity fluorescence sensors would be obtained.

In 2008, Yan et al. [33] reported the first index-guided SCF surface-enhanced Raman probe. The presence of gold nanoparticles served as a substrate of the surface-enhanced Raman scattering, which were either coated on the inner surface of the holes or mixed in the analytic solution. The numerical calculations predicted that an energy overlap as high as $21.45 \%$ could be reached.

Later on that year, Warren-Smith et al. [34] proposed the first theoretical study of the absorption and fluorescence sensing properties of the SCF when one of the air-holes was removed to expose the core to the external environment. One year later, the same group reported the new fabrication methods to obtain an exposed-core SCF. The response type for the exposed-core fiber was significantly higher than that for the enclosed core fiber [35].

In 2010, Oo et al. [36] reported the numerical simulation and hyperspectral Raman imaging of three index-guiding solid-core PCFs of different air-cladding microstructures. The SCF was more robust than the other fibers presented and had an overwhelming advantage in terms of the ultimate limit of detection of an analyte of interest. They achieved a detection sensitivity of $1 \times 10^{-10} \mathrm{M}$ R6G (Rhodamine 6G) in a sampling volume (about $7.3 \mu \mathrm{L}$ ) of an aqueous solution.

In 2011, Warren-Smith [37] presented the first SCF fluorescence sensor platform for aluminum ions using a surface-attached derivative of the lumogallion. Even though it was fabricated to detect $\mathrm{Al}^{3+}$ ions, it can also be applicable to the detection of other ions, such as $\mathrm{Ga}^{3+}$ and $\mathrm{In}^{3+}$. This sensor platform provided real-time, in situ sensing of $\mathrm{Al}^{3+}$ ions for biological applications and could also be applied to the corrosion sensing.

\subsection{Biological parameters}

In 2007, Ruan et al. [38] reported the use of the lead silica soft glass SCF to detect quantum-dot labeled proteins with near infrared light. Of the two types of glasses used, the $\mathrm{F} 2$ glass presented losses of $0.6 \mathrm{~dB} / \mathrm{m}$ in the visible and near infrared region, 
enabling the use of longer fibers for sensing. The detection limit achieved was of $1 \mathrm{nM}$ (nanomolar), using only $3 \%$ of the guided mode of the fiber. One year later, the same authors reported the immobilization of antibodies within the holes in the SCF cross-section [39]. For the first time, immobilized proteins have been detected within the soft glass SCF using fluorescence labeling techniques.

In 2010, Coscelli et al. [40] investigated the feasibility of a biosensor for DNA detection based on the SCF. By functionalizing the whole surface, the DNA strand binding was allowed. The probe described in this work was able to discriminate the DNA with only a single-base difference.

\section{Interferometry}

In this section, the work includes two types of interferometers. One of them is based on an FLM using two different geometries. The other is a multi-interferometer using a suspended seven-core fiber. Figure 2 presents the two typical geometries of the SCF based on four and three holes.

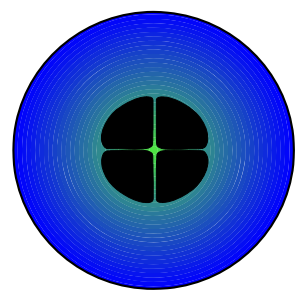

(a)

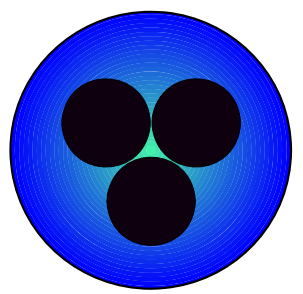

(b)
Fig. 2 Typical geometries of the suspended core fiber: (a) four holes and (b) three holes.

\subsection{Fiber loop mirror}

Figure 3 presents the fiber loop mirror interferometer configuration adapted to measure different physical parameters. It consists in a $3-\mathrm{dB}$ optical coupler with the low insertion loss, an optical polarization controller (PC), an optical source, and an optical spectrum analyzer (OSA). The sensing head was constituted by a section of the SCF spliced between the output ports of the optical coupler. Two different types of SCF were fabricated at the IPHT (Institute of Photonic Technology, Jena, Germany) and tested in the FLM configuration. The first SCF, with a section length of about $1.2 \mathrm{~m}$, was formed by four holes with a diameter of $43.3 \mathrm{~m}$. The core and the cladding had diameters of about $5.0 \mathrm{~m}$ and about $135.0 \mathrm{~m}$, respectively. This single core fiber was slightly elliptical owing to the hole asymmetry originated during the fabrication process. The second SCF used presented twin-core with a diameter of $1.5 \mu \mathrm{m}$, the cladding was $124 \mu \mathrm{m}$, and the big/small holes were $10 / 5 \mu \mathrm{m}$, respectively. A fiber section with a length of about $0.26 \mathrm{~m}$ was spliced.

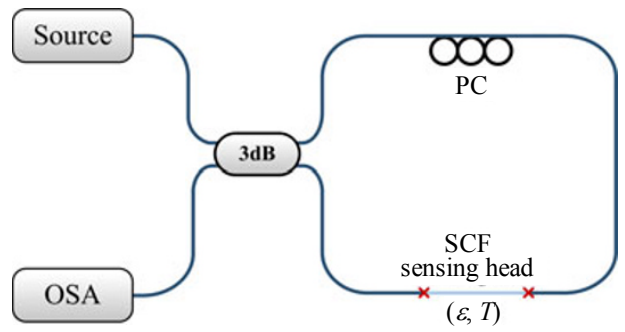

Fig. 3 Experimental configuration of the SCF-FLM.

Both fibers were characterized in strain and temperature. Figure 4 presents the responses of two sensing heads when strain is applied. In Table 1, the sensitivities of the both sensing heads are shown.

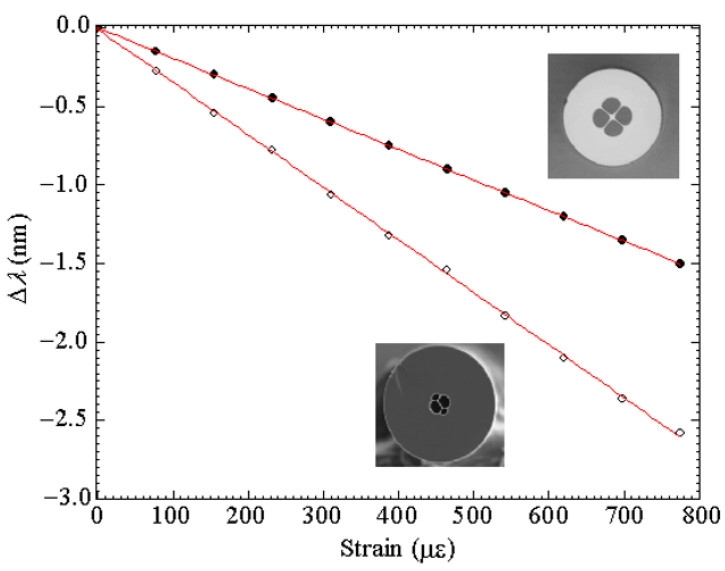

Fig. 4 Strain responses of two SCF-FLMs.

Table 1 Sensitivities of each sensing head.

\begin{tabular}{|c|c|c|}
\hline & Single core & Twin core \\
\hline Strain & $-1.94 \mathrm{pm} / \mu \varepsilon$ & $-3.35 \mathrm{pm} / \mu \varepsilon$ \\
\hline Temperature & $-0.29 \mathrm{pm} /{ }^{\circ} \mathrm{C}$ & $-20 \mathrm{pm} /{ }^{\circ} \mathrm{C}$ \\
\hline
\end{tabular}

The twin-core presents higher sensitivity due to the difference of the elasto-optic parameter between 
each core. Stress effects were produced in the cores during the manufacture, which gave rise to an optical path difference between the cores, when these were subjected to physical parameters.

Figure 5 shows the response of both sensing heads to temperature. When temperature increases, the stress created by manufacture is diminished.

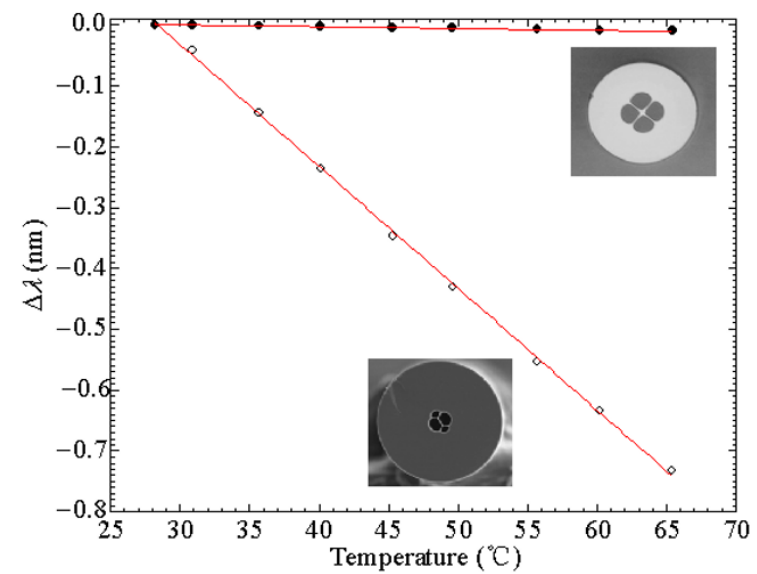

Fig. 5 Temperature responses of the two SCF-FLMs.

Comparing the two sensing heads, the single core shows evidence of being a good solution for a temperature-independent strain sensor. The twin core presents an alternative solution as a temperature independent torsion sensor using an analysis based on the amplitude signal converted in an FFT [13].

\subsection{Multi-interferometer}

The in-fiber multi-interferometer experimental configuration is presented in Fig. 6. A broadband optical source with a bandwidth of $100 \mathrm{~nm}$ and a central wavelength at $1550 \mathrm{~nm}$ was used to illuminate the multi-interferometer. An OSA was used to measure the transmission spectral response of the sensing head with a resolution of $0.1 \mathrm{~nm}$.

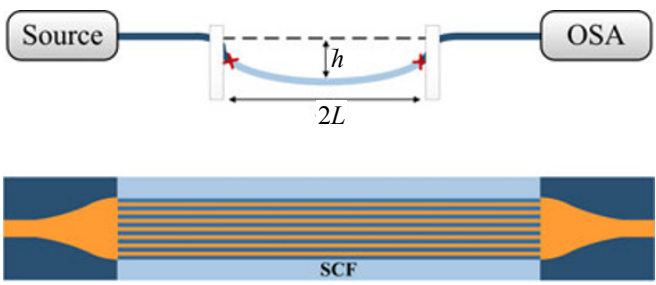

Fig. 6 Experimental configuration of the multi-interferometer and scheme of the sensing head.

Figure 7 presents the cross-section of the suspended multi-core fiber. Seven suspended cores constituted the fiber. The multi-core fiber section was spliced between two small sections of the expansible fiber (graded index) to guarantee not only the excitation of all cores, but also the coupling to the SMF [14]. In this situation, multi-interference would be expected in the second splice region. The configuration was analogous to a multiple Mach-Zehnder in parallel. The spectral response observed in the OSA was a modulated set of fringes with different amplitudes and frequencies, however, it could be processed when the FFT analysis was used [14].

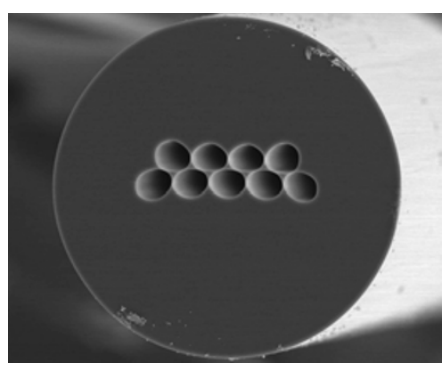

Fig. 7 Cross-section of the suspended multi-core fiber.

When the curvature was applied in this configuration, there was a change in the peak amplitude. The two peaks with the highest intensity were monitored, and the ratio between both signals exhibited a sensitivity of $-4.34 \mathrm{~dB} \cdot \mathrm{m}$, as shown in Fig. 8. This was a referenced analysis, resulting in the elimination of the source fluctuations. The

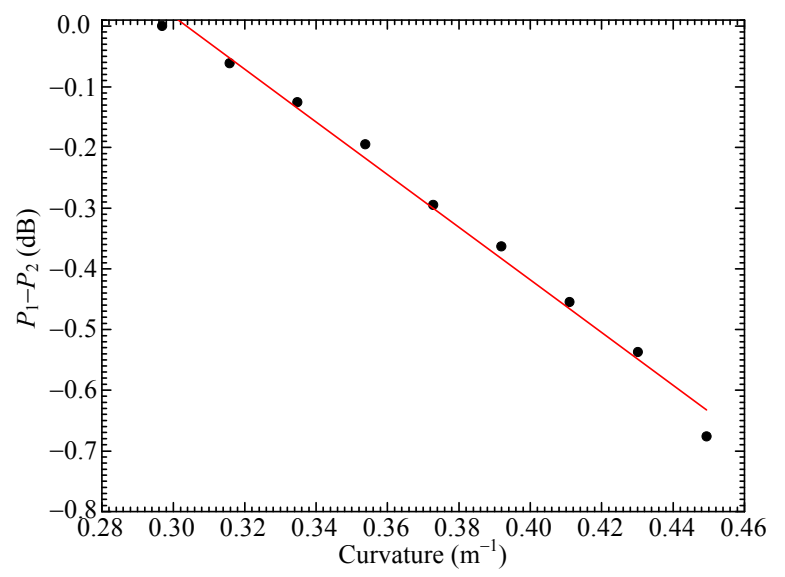

Fig. 8 Curvature response of the suspended multi-core fiber.

advantage of this configuration is that it can be used 
for simultaneous measurement of curvature and strain [14]. Relatively to the temperature sensing, the result is similar to the one reported in the literature [13].

\section{Future remark}

The SCF has been widely researched over the previous ten years. The first area where it was successfully explored was in gas sensing. However, it also proved to be good solution both in chemical and biological sensing. In these fields, future works include plasmonic and terahertz applications. The use of advanced materials in the fabrication, such as polymers, is also a matter of interest in order to obtain better performances.

Regarding physical parameters, the SCF can be a good solution for the enhancement of specific physical parameters, namely, pressure, curvature, and flow. Nevertheless, new geometries based on different hole structures creating new optical sensors with high precision and sensitivities can contribute for a new breakthrough. This development can be interesting not only in engineering, but also in medical applications where the small size of a sensing probe is crucial.

Open Access This article is distributed under the terms of the Creative Commons Attribution License which permits any use, distribution, and reproduction in any medium, provided the original author(s) and source are credited.

\section{References}

[1] T. M. Monro, W. Belardi, K. Furusawa, J. C. Baggett, N. G. R. Broderick, and D. J. Richardson, "Sensing with microstructured optical fibers," Meas. Sci. Technol., vol. 12, no. 7, pp. 854-858, 2001.

[2] A. S. Webb, F. Poletti, D. J. Richardson, and J. K. Sahu, "Suspended-core holey fiber for evanescent-field sensing," Opt. Eng., vol. 46, no. 1, 2007.

[3] W. N. MacPherson, E. J. Rigg, J. D. C. Jones,
V. V. R. K. Kumar, J. C. Knight, and P. S. Russell, "Finite-element analysis and experimental results for a microstructured fiber with enhanced hydrostatic pressure sensitivity," J. Lightwave Technol., vol. 23, no. 3, pp. 1227-1231, 2005.

[4] T. M. Monro, S. Warren-Smith, E. P. Schartner, A. Francois, S. Heng, H. Ebendorff-Heidepriem, and S. Afshar, "Sensing with suspended-core optical fibers," Opt. Fiber Technol., vol. 16, no. 6, pp. 343-356, 2010.

[5] L. B. Fu, B. K. Thomas, and L. Dong, "Efficient supercontinuum generations in silica suspended core fibers," Opt. Express, vol. 16, no. 24, pp. 19629-19642, 2008.

[6] I. Savelii, J. C. Jules, G. Gadret, B. Kibler, J. Fatome, M. El-Amraoui, N. Manikandan, X. Zheng, F. Desevedavy, J. M. Dudley, J. Troles, L. Brilland, G. Renversez, and F. Smektala, "Suspended core tellurite glass optical fibers for infrared supercontinuum generation," Opt. Mater., vol. 33, no. 11, pp. 1661-1666, 2011.

[7] A. M. R. Pinto, O. Frazao, J. L. Santos, M. Lopez-Amo, J. Kobelke, and K. Schuster, "Interrogation of a suspended-core Fabry-Perot temperature sensor through a dual wavelength Raman fiber laser," J. Lightwave Technol., vol. 28, no. 21, pp. 3149-3155, 2010.

[8] A. van Brakel, C. Grivas, M. N. Petrovich, and D. J. Richardson, "Micro-channels machined in microstructured optical fibers by femtosecond laser," Opt. Express, vol. 15, no. 14, pp. 8731-8736, 2007.

[9] F. M. Cox, R. Lwin, M. C. J. Large, and C. M. B. Cordeiro, "Opening up optical fibers," Opt. Express, vol. 15, no. 19, pp. 11843-11848, 2007.

[10] H. Ebendorff-Heidepriem, S. C. Warren-Smith, and T. M. Monro, "Suspended nanowires: fabrication, design and characterization of fibers with nanoscale cores," Opt. Express, vol. 17, no. 4, pp. 2646-2657, 2009.

[11] O. Frazao, J. M. Baptista, J. L. Santos, J. Kobelke, and K. Schuster, "Strain and temperature characterisation of sensing head based on suspended-core fiber in Sagnac interferometer," Electron. Lett., vol. 44, no. 25, pp. 1455-1456, 2008.

[12] A. Ryasnyanskiy, A. X. Lin, A. Belwalkar, C. Guintrand, I. Biaggio, and J. Toulouse, "Nonlinear frequency conversion in bismuth-doped tellurite suspended core fiber," Opt. Commun., vol. 284, no. 16-17, pp. 3977-3979, 2011.

[13] O. Frazao, R. M. Silva, J. Kobelke, and K. Schuster, "Temperature- and strain-independent torsion sensor using a fiber loop mirror based on suspended twin-core fiber," Opt. Lett., vol. 35, no. 16, pp. 2777-2779, 2010. 
[14] R. M. Silva, M. S. Ferreira, J. Kobelke, K. Schuster, and O. Frazao, "Simultaneous measurement of curvature and strain using a suspended multicore fiber," Opt. Lett., vol. 36, no. 19, pp. 3939-3941, 2011.

[15] M. Roze, B. Ung, A. Mazhorova, M. Walther, and M. Skorobogatiy, "Suspended core subwavelength fibers: towards practical designs for low-loss terahertz guidance," Opt. Express, vol. 19, no. 10, pp. 9127-9138, 2011.

[16] M. C. P. Huy, G. Laffont, V. Dewynter, P. Ferdinand, P. Roy, J. L. Auguste, D. Pagnoux, W. Blanc, and B. Dussardierg, "Three-hole microstructured optical fiber for efficient fiber Bragg grating refractometer," Opt. Lett., vol. 32, no. 16, pp. 2390-2392, 2007.

[17] M. Hautakorpi, M. Mattinen, and H. Ludvigsen, "Surface-plasmon-resonance sensor based on three-hole microstructured optical fiber," Opt. Express, vol. 16, no. 12, pp. 8427-8432, 2008.

[18] J. Wojcik, P. Mergo, M. Makara, K. Poturaj, L. Czyzewska, J. Klimek, and A. Walewski, "Technology of suspended core microstructured optical fibers for evanesced wave and plasmon resonance optical fiber sensors," in Proc. of Photonic Crystal Fibers II, Strasbourg, April 9-10, pp. 69900T-1-69900T-6, 2008.

[19] S. Torres-Peiró, A. Díez, J. L. Cruz, and M. V. Andrés, "Temperature sensor based on Ge-doped microstructured fibers," Journal of Sensors, vol. 2009, pp. 417540-1-417540-5, 2009.

[20] B. Gauvreau, F. Desevedavy, N. Guo, D. Khadri, A. Hassani, and M. Skorobogatiy, "High numerical aperture polymer microstructured fiber with three super-wavelength bridges," J. Opt. A-Pure Appl. Op., vol. 11, no. 8, pp. 85102-85110, 2009.

[21] O. Frazao, S. H. Aref, J. M. Baptista, J. L. Santos, H. Latifi, F. Farahi, J. Kobelke, and K. Schuster, "Fabry-Perot cavity based on a suspended-core fiber for strain and temperature measurement," IEEE Photonics Tech. Lett., vol. 21, no. 17, pp. 1229-1231, 2009.

[22] O. Frazao, J. M. Baptista, J. L. Santos, J. Kobelke, and K. Shuster, "Refractive index tip sensor based on Fabry-Perot cavities formed by a suspended core fiber," J. Eur. Opt. Soc-Rapid, vol. 4, pp. 28-31, 2009.

[23] O. Frazao, S. F. O. Silva, J. Viegas, J. M. Baptista, J. L. Santos, J. Kobelke, and K. Schuster, "All fiber Mach-Zehnder interferometer based on suspended twin-core fiber," IEEE Photonics Tech. Lett., vol. 22, no. 17, pp. 1300-1302, 2010.

[24] S. F. O. Silva, J. L. Santos, J. Kobelke, K. Schuster, and O. Frazao, "Simultaneous measurement of three parameters using an all-fiber Mach-Zehnder interferometer based on suspended twin-core fibers," Opt. Eng., vol. 50, no. 3, pp. 030501-1-030501-3, 2011.

[25] C. M. Jewart, T. Chen, E. Lindner, J. Fiebrandt, M. Rothhardt, K. Schuster, J. Kobelke, H. Bartelt, and K. P. Chen, "Suspended-core fiber Bragg grating sensor for directional-dependent transverse stress monitoring," Opt. Lett., vol. 36, no. 12, pp. 2360-2362, 2011.

[26] M. Bravo, A. Pinto, M. Lopez-Amo, J. Kobelke, and K. Schuster, "High precision micro-displacement fiber sensor through a suspended-core Sagnac interferometer," Opt. Lett., vol. 37, no. 2, pp. 202-204, 2012.

[27] S. H. Aref, M. I. Zibaii, M. Kheiri, H. Porbeyram, H. Latifi, F. M. Araujo, L. A. Ferreira, J. L. Santos, J. Kobelke, K. Schuster, and O. Frazao, "Pressure and temperature characterization of two interferometric configurations based on suspended-core fibers," Opt. Commun., vol. 285, no. 3, pp. 269-273, 2012.

[28] C. M. B. Cordeiro, C. J. S. de Matos, E. M. dos Santos, A. Bozolan, J. S. K. Ong, T. Facincani, G. Chesini, A. R. Vaz, and C. H. B. Cruz, "Towards practical liquid and gas sensing with photonic crystal fibers: side access to the fiber microstructure and single-mode liquid-core fiber," Meas. Sci. Technol., vol. 18, no. 10, pp. 3075-3081, 2007.

[29] T. G. Euser, J. S. Y. Chen, M. Scharrer, P. S. J. Russell, N. J. Farrer, and P. J. Sadler, "Quantitative broadband chemical sensing in air-suspended solid-core fibers," J. Appl. Phys., vol. 103, no. 10, pp. 103108-1-103108-7, 2008.

[30] H. Lehmann, J. Kobelke, K. Schuster, R. Willsch, H. Bartelt, R. Amezcua-Correa, and J. C. Knight, "Gas sensing with suspended core fibers and hollow core band gap fibers: a comparative study," in Proc. SPIE (20th International Conference on Optical Fiber Sensors), Edinburgh, October 5-9, vol. 7503, pp. 75035C, 2009.

[31]S. Selleri, E. Coscelli, M. Sozzi, A. Cucinotta, F. Poli, and D. Passaro, "Air-suspended solid-core fibers for sensing," in Proc. SPIE (Optical Sensors 2009), Prague, April 20-22, vol. 7536, pp. 73561L, 2009.

[32] S. Afshar, S. C. Warren-Smith, and T. M. Monro, "Enhancement of fluorescence-based sensing using microstructured optical fibers," Opt. Express, vol. 15, no. 26, pp. 17891-17901, 2007.

[33] H. Yan, J. Liu, C. X. Yang, G. F. Jin, C. Gu, and L. Hou, "Novel index-guided photonic crystal fiber surface-enhanced Raman scattering probe," Opt. Express, vol. 16, no. 11, pp. 8300-8305, 2008.

[34] S. C. Warren-Smith, S. Afshar, and T. M. Monro, "Theoretical study of liquid-immersed exposed-core 
microstructured optical fibers for sensing," Opt. Express, vol. 16, no. 12, pp. 9034-9045, 2008.

[35] S. C. Warren-Smith, H. Ebendorff-Heidepriem, T. C. Foo, R. Moore, C. Davis, and T. M. Monro, "Exposed-core microstructured optical fibers for real-time fluorescence sensing," Opt. Express, vol. 17, no. 21, pp. 18533-18542, 2009.

[36] M. K. K. Oo, Y. Han, J. Kanka, S. Sukhishvili, and H. Du, "Structure fits the purpose: photonic crystal fibers for evanescent-field surface-enhanced Raman spectroscopy," Opt. Lett., vol. 35, no. 4, pp. 466-468, 2010.

[37] S. C. Warren-Smith, S. Heng, H. EbendorffHeidepriem, A. D. Abell, and T. M. Monro, "Fluorescence-based aluminum ion sensing using a surface-functionalized microstructured optical fiber," Langmuir, vol. 27, no. 9, pp. 5680-5685, 2011.
[38] Y. L. Ruan, E. P. Schartner, H. EbendorffHeidepriem, P. Hoffmann, and T. M. Monro, "Detection of quantum-dot labeled proteins using soft glass microstructured optical fibers," Opt. Express, vol. 15, no. 26, pp. 17819-17826, 2007.

[39] Y. Ruan, T. C. Foo, S. Warren-Smith, P. Hoffmann, R. C. Moore, H. Ebendorff-Heidepriem, and T. M. Monro, "Antibody immobilization within glass microstructured fibers: a route to sensitive and selective biosensors," Opt. Express, vol. 16, no. 22, pp. 18514-18523, 2008.

[40] E. Coscelli, M. Sozzi, F. Poli, D. Passaro, A. Cucinotta, S. Selleri, R. Corradini, and R. Marchelli, "Toward A Highly Specific DNA Biosensor: PNA-Modified Suspended-Core Photonic Crystal Fibers," IEEE J. Sel. Top. Quant., vol. 16, no. 4, pp. 967-972, 2010. 